\title{
Temporarily Unavailable
}

National Cancer Institute

\section{Source}

National Cancer Institute. Temporarily Unavailable. NCI Thesaurus. Code C150903.

Information is not available currently but expected to be made available in the near future. 